\title{
Criteria for Distinguishing Floc Sedimentation and Gel-Like Network Sedimentation of Cohesive Fine-Grained Sediment in a Turbulent Flow
}

\author{
Zhongfan Zhu \\ College of Water Sciences, Beijing Normal University, Beijing, China \\ Email: zhuzhongfan1985@gmail.com
}

Received November 2014

\begin{abstract}
The study on the criteria used to distinguish floc sedimentation and gel-like network sedimentation of cohesive fine-grained sediment in a turbulent flow has rarely been carried out. In this preliminary study, we derive three criteria to distinguish these two different sedimentation phases by considering the comparison of the time that a characteristic floc takes to settle through mean distance between characteristic flocs and the time that it takes to move through the same distance under the influence of a turbulent flow. These criteria incorporate physical and chemical factors that have been verified to have some influences on the sediment flocculation in some published literatures, and a simple analysis result is found to be consistent with our qualitative understandings of flocculation phenomenon of cohesive fine-grained sediment.
\end{abstract}

\section{Keywords}

Criteria, Floc Sedimentation, Gel-Like Network Sedimentation, Cohesive Sediment, Turbulent Flow

\section{Introduction}

Under influences of some physical and chemical conditions, cohesive fine-grained sediment particles charged with ion on their surfaces can get into contact, and further coagulate because of the Brownian diffusion, a flow shear and a differential sedimentation, consequently forming large number of flocs or a gel-like network structure (this kind of structure is composed of flocs contacting with each other, and spans total sediment suspension system) (Yang et al., 2003). This process has been termed as flocculation. As Zhu et al. (2009) have pointed out, single particle, flocs and gel-like network can be regarded to be three different degrees of flocculation development in a sediment suspension system, and as a result we can define three flocculation phases of cohesive sediment as follows: single-particle sedimentation phase, floc sedimentation phase and gel-like network sedimentation phase. Furthermore, Zhu et al. (2009) presented that it was of vital importance to put forward two criteria for describing the transition between single-particle sedimentation phase and floc sedimentation phase and that 
between floc sedimentation phase and gel-like network sedimentation phase, because there will be entirely different methods for calculating dynamic characteristic of sediment particle in the suspension system belonging to different phase. Between two criteria, they further indicated that the first criteria (i.e., the criteria between single-particle sedimentation phase and floc sedimentation phase) had been commonly regarded to be particle size or concentration of electrolyte in the suspension to a large degree, and study on the rest criteria may need to be paid great attention. Based on their preliminary study, we attempt to present the criteria for distinguishing floc sedimentation phase and gel-like network sedimentation phase of cohesive sediment in a turbulent flow in this study.

Yang et al. (2003) summarized all of the factors that have been verified to have effects on the flocculation of cohesive sediment. These factors can be concluded to be three kinds of factors:

(1) Physical factors: mineral composition of sediment particle, radius of primary particle, $a$, volumetric concentration of primary particle, $\phi$, mass density of primary particle, $\rho_{s}$, and temperature, $T$;

(2) Chemical factors: concentration of electrolyte, $n$, in the suspension, valence of cation of electrolyte, $z$;

(3) Mechanical factors: the Brownian motion, a differential sedimentation and a flow shear.

These factors interact mutually in the suspension, making the sediment flocculation in the suspension system to exhibit different phases. For the transition between floc sedimentation phase and gel-like network sedimentation phase, particularly the criteria in a turbulent flow, there are few studies. Fei (1994) suggested to use solid surface area per volume of the suspension as the criteria to distinguish floc sedimentation and gel-like network sedimentation in a quiescent sediment suspension. However, these criteria are somewhat empirical, not considering effects of all kinds of factors. A feasible method for establishing criteria is to construct an non-dimensional parameter by comprehensively considering all of physical, chemical and mechanic factors that can influence the flocculation process.

\section{Derivation of Criteria}

For the sake of analyzing the flocculation process of cohesive sediment in a turbulent flow, we adopted concepts of "characteristic floc" and critical condition of occurrence of a gel-like network structure both put forward by Zhu et al. (2009) as follows: we simply consider that the maximum size of floc in a turbulent flow is a specific value for a given sediment suspension system. Those flocs whose sizes are equal to the maximum size are regarded as "characteristic flocs". Intensifying the factors that can provoke the process of flocculation in the sediment suspension system could result in a increasing number of characteristic flocs. When number of characteristic flocs in the suspension is enough large, these characteristic flocs can get into contact, subsequently forming a gel-like network that spans the entire suspension system, as schematically shown in Figure 1. Moreover, we roughly define the concept of critical condition of occurrence of a gel-like network as follows: for a specific sediment suspension system, as long as those factors that can promote the flocculation process (such as concentration of electrolyte in the suspension, cation of electrolyte, volumetric concentration of primary particle) reaches some critical values respectively, the speed of occurrence of a gel-like network is so rapid that those characteristic flocs in the suspension system can gather quickly together and subsequently construct a gel-like network rather than settling individually. This condition is simply regarded to be critical condition of

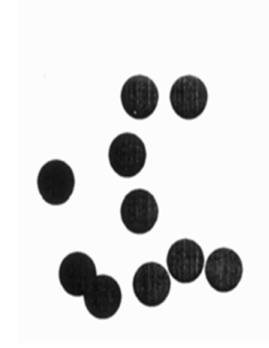

Primary particle

(a)

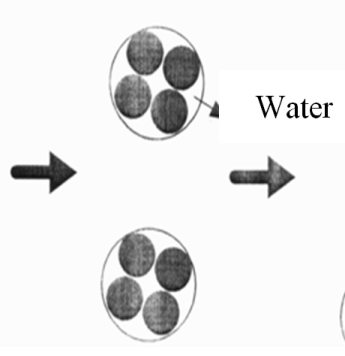

Characteristic floc

(b)

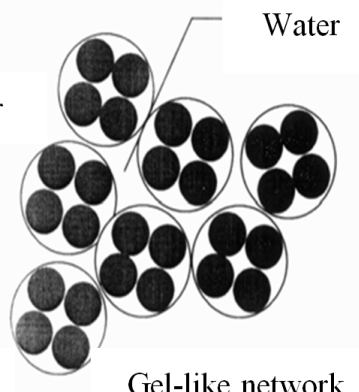

(c)

Figure 1. Three phases of flocculation development of cohesive sediment particle in the sediment suspension system: (a) single particle sedimentation; (b) floc sedimentation; (c) gel-like network sedimentation (Zhu et al., 2009). 
occurrence of gel-like network.

Floc can be described as a fractal object, and the relation between size of fractal object, $d_{f}$, and number of single particle combined in this floc, $N$, was expressed in Feder (1988) as follows:

$$
N=\left(\frac{d_{f}}{2 a}\right)^{\mathrm{D}},
$$

where $a$ is radius of primary particle as already introduced, $D$ fractal dimension of floc, $d_{f}$ size of floc. In a turbulent flow, breakup of floc should result from a flow shear. When a hydrodynamic shear stress exerted on the floc exceeds strength of foc, breakup of floc may happen. Thus, for the characteristic floc, its critical strength, $\sigma_{T}$, should be equal to the hydrodynamic stress, $\tau$, exerted by the turbulent flow on this characteristic floc, that is

$$
\sigma_{T}=\tau \text {. }
$$

For a large floc in inertial range of the turbulent flow $\left(d_{f} \gg \eta\right.$, here $\eta$ is Kolmogrov scale in the turbulent flow, $\eta=\left(v^{3} / \varepsilon\right)^{1 / 4}$, where $\varepsilon$ is energy dissipation rate, and $v$ the kinematic viscosity of fluid), floc strength can be gotten as expressed in Lu et al. (1998) as follows:

$$
\sigma_{T} \approx \frac{F}{d_{f}(2 a)^{2}}\left(\frac{v^{3}}{\varepsilon}\right)^{\frac{1}{4}}
$$

whereas for a small floc $\left(d_{f}<\eta\right)$, strength of the floc can be expressed as also presented in Lu et al. (1998) as follows:

$$
\sigma_{T} \approx \frac{F}{d_{f}^{2}} .
$$

In both of Equation (3) and Equation (4), $F$ is the comprehensive force presented in DLVO theory, $F=F_{A}+F_{R}, \quad F_{A}=-\frac{A_{131} a}{24 H^{2}}, \quad F_{R}=\frac{16 \pi \varepsilon a K_{B}^{2} T^{2}}{e^{2} z^{2} H}\left(\frac{\exp \left(z e \psi / 2 K_{B} T\right)-1}{\exp \left(z e \psi / 2 K_{B} T\right)+1}\right)^{2} \exp (-\kappa H), F_{A}$ van der Waals attraction force, $F_{R}$ the electrical double layer repulsion force, $A_{131}$ the effective Hamaker constant, $H$ distance between surface of primary particle, $\varepsilon$ permittivity, $K_{B}$ Boltzmann constant, $T$ temperature as introduced, $e$ electric quantity of electron, $z$ valence of cation of electrolyte as already introduced, $\psi$ surface potential of particle, $\kappa^{-1}$ thickness of electrical double layer $\left(\kappa=\sqrt{2 n z^{2} e^{2} / \varepsilon K_{B} T}\right)$.

When floc is in viscosity range of the turbulent flow $\left(d_{f}<2 \sim 3 \eta\right.$ ), hydrodynamic shear stress on the floc can be gotten in Lu et al. (1998) as follows:

$$
\tau=0.26 \rho_{w}(v \varepsilon)^{\frac{1}{2}}
$$

where $\rho_{w}$ is the mass density of fluid. While for the floc in transition range or inertial range of the turbulent flow ( $d_{f} \gg 25 \eta$ ), hydrodynamics shear stress can be expressed as also found in Lu et al. (1998):

$$
\begin{gathered}
\text { in transition range: } \tau=0.49 \rho_{w}\left(\frac{\varepsilon^{3}}{v}\right)^{\frac{1}{4}} d_{f}, \\
\text { in inertial range: } \tau=1.9 \rho_{w}\left(\varepsilon d_{f}\right)^{\frac{2}{3}} .
\end{gathered}
$$

Substituting Equation (3)-Equation (7) into Equation (2) can get different expressions of size, $d_{f \max }$, of characteristic floc in different ranges as follows:

$$
\text { in viscous range: } d_{f \max }=2 \rho_{w}^{-\frac{1}{2}}(\varepsilon v)^{-\frac{1}{4}} F^{\frac{1}{2}} \text {, }
$$




$$
\begin{aligned}
& \text { in transition range: } d_{f \max }=\frac{1.5 F^{\frac{1}{2}}}{\rho_{w}^{\frac{1}{2}}(2 a)}\left(\frac{\varepsilon}{v}\right)^{-\frac{1}{2}}, \\
& \text { in inertial range: } d_{f \max }=\left[\frac{F v^{\frac{3}{4}}}{1.9 \rho_{w} \varepsilon^{\frac{11}{12}}(2 a)^{2}}\right]^{\frac{3}{5}} .
\end{aligned}
$$

When a large number of characteristic flocs have been presented in a turbulent flow, there are two types of sedimentation that these flocs may choose. One is a individual sedimentation: these characteristic flocs can settle individually, and the suspension system undergoes floc sedimentation; The other is a gel-like network collective sedimentation due to a flow shear rather than the Brownian diffusion (compared to a flow shear, effect of the Brownian motion can be neglected): these characteristic flocs can get into contact, and further construct a network structure, hence the suspension undergoes the network collective sedimentation. Thus the relative importance of flow shear and individual settling decides the sedimentation condition to which the sediment suspension system corresponds: network sedimentation or floc sedimentation. A seemingly feasible method to estimate the relative importance of flow shear and individual settling is to compare the time, $t_{s}$, that a characteristic floc takes to settle through mean distance between characteristic flocs and the time, $t_{f}$, that it takes to move through the same distance due to the flow shear.

From Equation (1), volumetric concentration of characteristic floc, $\phi_{f}$, in the suspension can be gotten to be $\phi\left(d_{f \max } / 2 a\right)^{3-D}$. The average distance between characteristic flocs should be $\left(d_{f \max } / 2\right) \phi^{-1 / 3}\left(d_{f \max } / 2 a\right)^{D / 3-1}$. By considering a diffusivity coefficient of characteristic floc in a turbulent flow (a detailed derivation can be found in Zhu (2009)), the time, $t_{f}$, that characteristic floc takes to move through the average distance between characteristic flocs due to a flow shear can be obtained as follows

$$
t_{f}=\frac{\left[\frac{d_{f \max }}{2} \phi^{-\frac{1}{3}}\left(\frac{d_{f \max }}{2 a}\right)^{\frac{D}{3}-1}\right]^{2}}{\left(\frac{\varepsilon}{v}\right)^{\frac{1}{2}} d_{f \max }^{2}}=\frac{\phi^{-\frac{2}{3}}}{4\left(\frac{\varepsilon}{v}\right)^{1 / 2}(2 a)^{\frac{2 D}{3}-2}} d_{f \max }^{\frac{2 D}{3}-2},
$$

when characteristic floc is in viscous range of the turbulent flow, and

$$
t_{f}=\frac{\left[\frac{d_{f \max }}{2} \phi^{-\frac{1}{3}}\left(\frac{d_{f \max }}{2 a}\right)^{\frac{D}{3}-1}\right]^{2}}{\varepsilon^{\frac{1}{3}} d_{f \max }^{\frac{4}{3}}}=\frac{\phi^{-\frac{2}{3}}}{4 \varepsilon^{\frac{1}{3}}(2 a)^{\frac{2 D}{3}-2}} d_{f \text { max }}^{\frac{2 D-4}{3}},
$$

when characteristic floc is in transition range or inertial range.

On the other hand, settling velocity, $\omega$, of characteristic floc can be found in Wang et al. (2002) as follows:

$$
\omega=1.72 \sqrt{\frac{\Delta \rho}{\rho_{w}} g d_{f \max }},
$$

where $\Delta \rho=\rho_{s}-\rho_{w}$, and $g$ a gravitation acceleration. Thus, the time, $t_{s}$, can be gotten with Equation (13) and the average distance between characteristic flocs in the following expression:

$$
t_{s}=\frac{\phi^{-\frac{1}{3}}}{2}\left(\frac{1}{2 a}\right)^{\frac{D}{3}-1} \frac{1}{1.72 \sqrt{\frac{\Delta \rho g}{\rho_{w}}}}\left(d_{f \max }\right)^{\frac{2 D-3}{6}} .
$$


If $t_{s}>t_{f}$, these characteristic flocs can get into contact due to a flow shear, finally forming a gel-like structure that spans total suspension system. Whereas for $t_{s}<t_{f}$, sedimentation of characteristic floc begins before the gel-like network is presented, and characteristic floc should settle individually. Thus, the critical condition between floc sedimentation phase and gel-like network sedimentation phase in the sediment suspension system can be gained by simply considering $t_{s}=t_{f}$.

When characteristic floc is in viscosity range of the turbulent flow, an non-dimensional parameter can be gotten by combining Equation (8), Equation (11) and Equation (14) as follows:

$$
P_{\text {tur } 1}=\frac{(2 a)^{\frac{5 D}{6}-\frac{5}{2}} \times 2^{4-\frac{5 D}{6}}}{1.72} \frac{1}{\sqrt{\left(\rho_{s}-\rho_{w}\right) g}} \rho_{w}^{-1+\frac{5 D}{12}} \phi^{1 / 3} G^{\frac{5 D}{12}-\frac{1}{2}} v^{-\frac{3}{2}+\frac{5 D}{12}} F^{\frac{3}{2}-\frac{5 D}{12}} \text {, where } G \text { is velocity gradient of the }
$$

turbulent flow, here $G=(\varepsilon / v)^{1 / 2}$ (Camp \& Stein, 1943). If $P_{\text {tur } 1}>1$, the suspension system undergoes gel-like network sedimentation; otherwise the system undergoes floc sedimentation.

When characteristic floc is in transition range, combination of Equation (9), Equation (12) and Equation (14) can have an non-dimensional parameter:

$$
P_{\text {tur } 2}=\frac{(1.5)^{\frac{14-5 D}{6}}}{0.86 \sqrt{\left(\rho_{s}-\rho_{w}\right) g}}(2 a)^{\frac{10 D-29}{6}} \rho_{w}^{\frac{5 D-8}{12}} \phi^{\frac{1}{3}} G^{\frac{5 D-10}{6}} v^{\frac{1}{3}} F^{\frac{14-5 D}{12}} .
$$

As long as $P_{\text {tur } 2}>1$, the suspension system is in gel-like network sedimentation phase; otherwise the system is in floc sedimentation phase.

When characteristic floc is in inertial range, an non-dimensional parameter can be obtained with Equation (10), Equation (12) and Equation (14) as follows

$$
P_{\text {tur } 3}=\frac{1}{1.9^{\frac{42-15 D}{30}} \times 0.86 \sqrt{\left(\rho_{s}-\rho_{w}\right) g}}(2 a)^{\frac{11}{6} D-\frac{159}{30}} \rho_{w}^{\frac{15 D-27}{30}} \phi^{\frac{1}{3}} G^{\frac{165 D-342}{180}} v^{\frac{30 D-4}{360}} F^{\frac{42-15 D}{30}} .
$$

Only if $P_{\text {tur }}>1$, the suspension system is in gel-like network sedimentation phase; for $P_{\text {tur } 3}<1$, the system should be in floc sedimentation phase.

\section{Simple Discussion}

In terms of the form, these three non-dimensional parameters embody all of the factors that can have some influences on the flocculation of cohesive sediment (such as radius of primary particles, volumetric concentration of primary particle, mass density of primary particle, concentration of electrolyte and valence of cation of electrolyte (these parameters are contained in the parameter $F$ ), velocity gradient of the turbulent flow).

Consider a typical example: radius of primary particle $a$ is $6.95 \mu \mathrm{m}$, mass density of primary particle $\rho_{\mathrm{s}}$ is $2650 \mathrm{~kg} / \mathrm{m}^{3}$, kinematical viscosity of water $v$ is $10^{-6} \mathrm{~m}^{2} / \mathrm{s}$, density of fluid $\rho_{w}$ is $1000 \mathrm{~kg} / \mathrm{m}^{3}$, and a typical value of fractal dimension of the characteristic floc $D$ is 2.5 .

Figures 2(a)-(c) show phases of flocculation in the sediment suspension system in the case of velocity gradient $G=100 \mathrm{~s}^{-1}$, when characteristic floc is in viscous range, transition range and inertial range of the turbulent flow, respectively. The horizontal axe denotes condition of electrolyte in the suspension system (Each $F$ value corresponds to a condition of electrolyte in the suspension: if either the concentration of electrolyte, $n$, or valence of cation of electrolyte, $z$, is increased (or both are increased), $F$ value can increase accordingly), and the vertical axe represents volumetric concentration of primary particle in the suspension system. It can be found that the line $P_{\text {tur } 1}=1$ or $P_{\text {tur } 2}=1$ or $P_{\text {tur } 3}=1$ divided the entire area into two different areas: the upper-right area is the region in which the suspension system is in gel-like sedimentation phase, and the lower-left part is the one where the system is in floc sedimentation phase. It can be found that if $F$ value is improved (i.e., condition of electrolyte is intensified) critical volume concentration needed for occurrence of a gel-like network can be reduced accordingly. Figures 3(a)-(c) show phases of flocculation in the sediment suspension system corresponding to different velocity gradients of the turbulent flow, when characteristic floc is in viscous range, transition range and inertial range, respectively. It can be readily found that the large velocity 


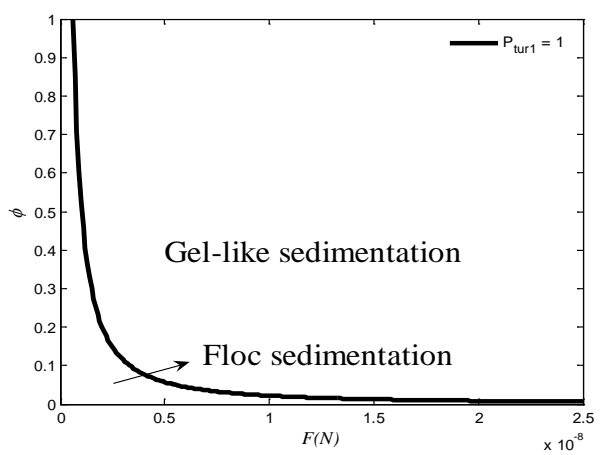

(a)

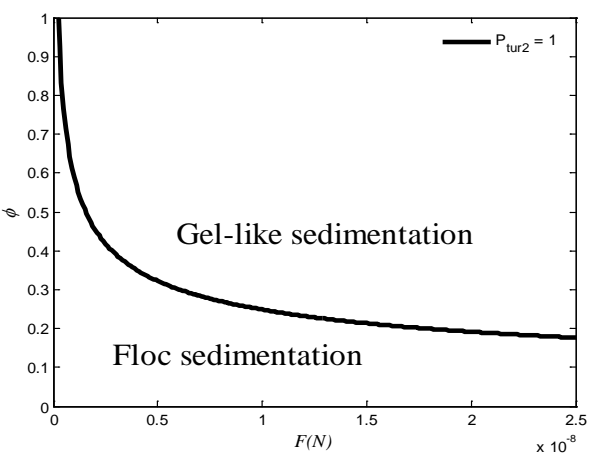

(b)

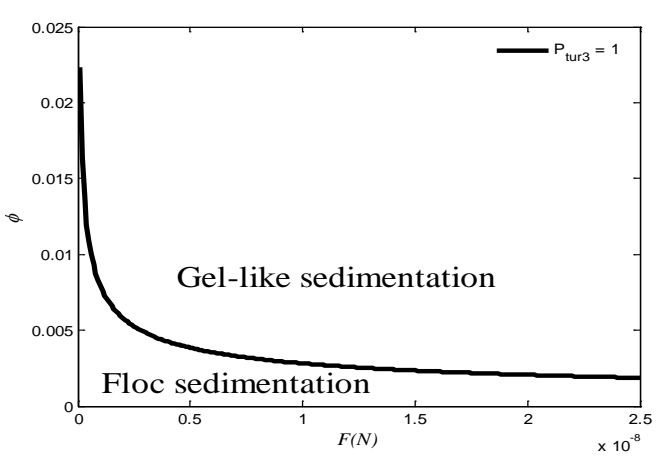

(c)

Figure 2. Phases of flocculation of cohesive fine-grained sediment in the suspension system when the characteristic floc is in the viscous range (a); the transition range (b) and the inertial range (c) of the turbulent flow, respectively.

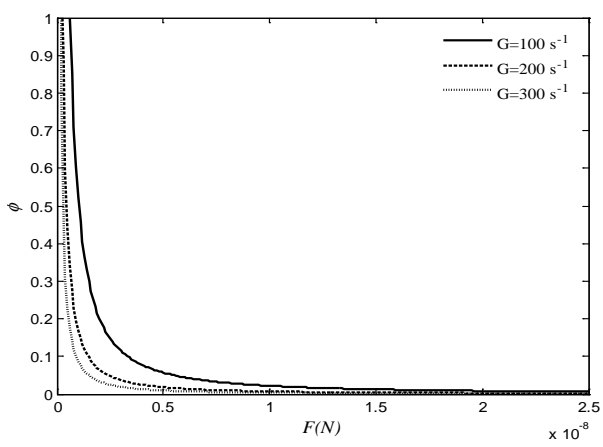

(a)

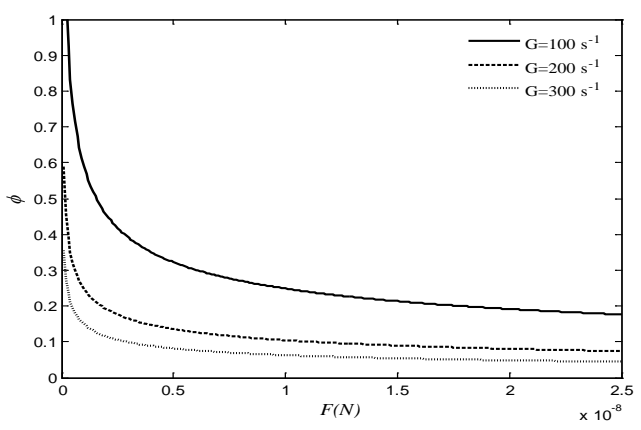

(b)

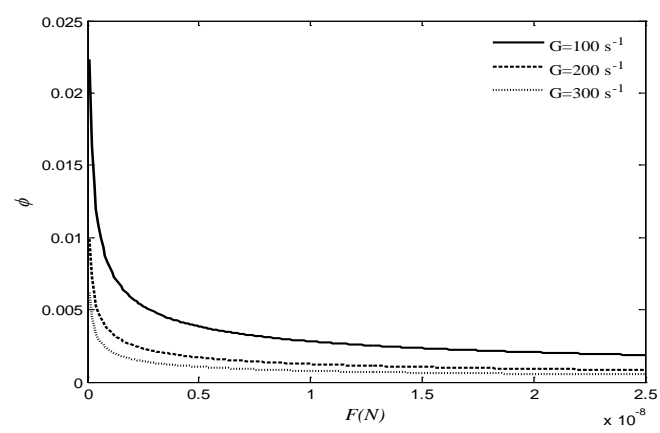

(c)

Figure 3. Phases of flocculation in the sediment suspension system corresponds to different velocity gradients of the turbulent flow, when the characteristic floc is in the viscous range (a); the transition range (b) and the inertial range (c), respectively. 
gradient of the turbulent flow corresponds to a larger area of occurrence of gel-like network. This indicates that a large shear flow increase collision frequency between flocs, consequently promoting the development of sediment flocculation. Figure 4(a)-(c) show phases of flocculation in the sediment suspension system corresponding to primary sediment particle with a different mass density in the case of velocity gradient $G=100 \mathrm{~s}^{-1}$, when characteristic floc is in viscous range, transition range and inertial range, respectively. It can be observed that the large mass density of primary sediment particle corresponds to a small area of occurrence of gel-like network. This is because characteristic flocs consisted of several primary particles with a large mass density have the larger gravities, and it is prone for them in the sediment suspension system to settle individually, there by making the suspension system tend to be in floc sedimentation phase. These conclusions seem to agree with our qualitative understandings of flocculation phenomenon of cohesive fine-grained sediment.

\section{Concluding Remarks}

Three non-dimensional parameters $P_{t u r 1}, P_{t u r 2}, P_{t u r 3}$ have been derived to distinguish floc sedimentation and gel-like network sedimentation of cohesive fine-grained sediment in a turbulent flow by comparing the time that a characteristic floc takes to settle through mean distance between characteristic flocs and the time that it takes to move through the same distance due to a flow shear. These three parameters corresponds to three different conditions where characteristic floc in the suspension system is in viscous range, transition range and inertial range of the turbulent flow respectively. When $P_{t u r 1}$ or $P_{t u r 2}$ or $P_{t u r 3}$ is larger than unity respectively, the sediment suspension system undergoes gel-like network sedimentation, otherwise it is in floc sedimentation phase. These parameters incorporate physical and chemical factors that have been verified to have some influences on

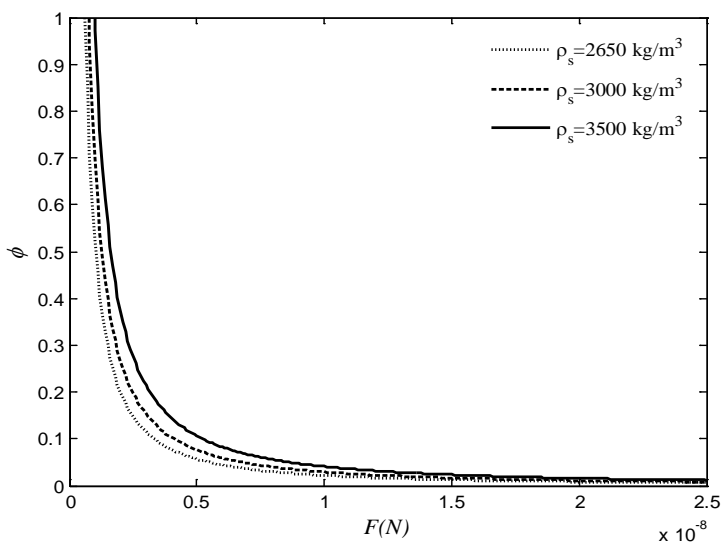

(a)

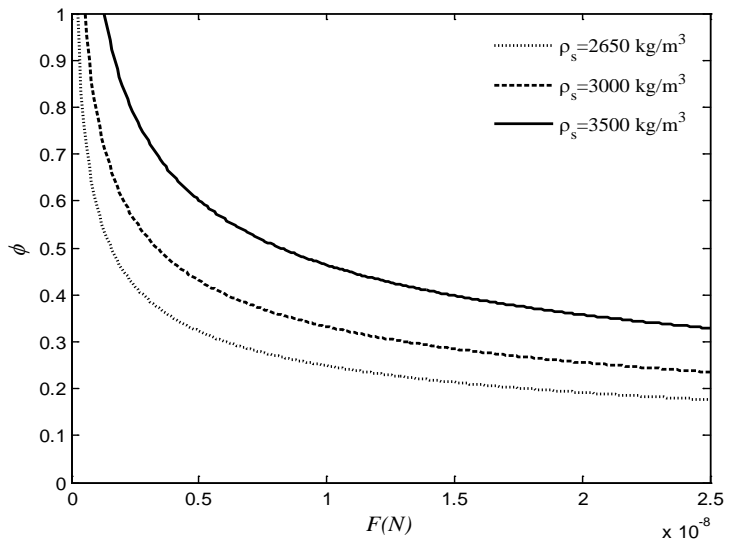

(b)

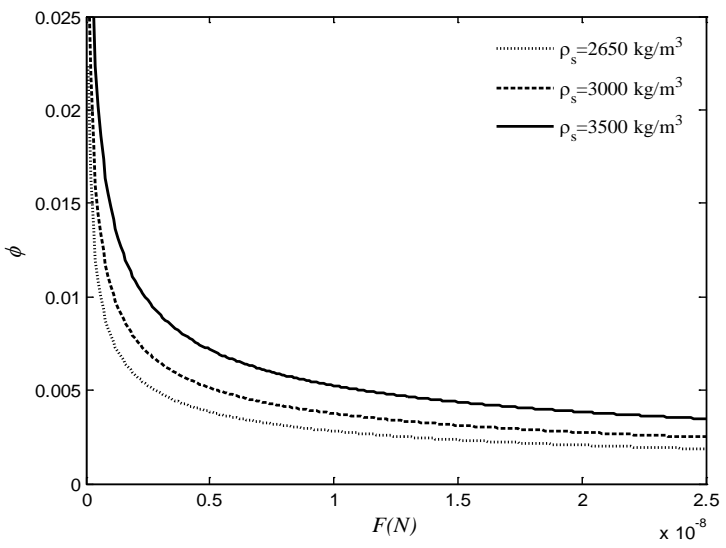

(c)

Figure 4. Phases of flocculation in the sediment suspension system corresponds to primary sediment particle with a different mass density, when the characteristic floc is in the viscous range (a); the transition range (b) and the inertial range (c) of the turbulent flow, respectively. 
the sediment flocculation in some published literatures, and a simple analysis result is found to be qualitatively consistent with our understandings of flocculation phenomenon of cohesive fine-grained sediment.

\section{Acknowledgements}

This research is supported by the Fundamental Research Funds for the Central Universities in China (Number: 2013NT50).

\section{References}

Camp, T. R., \& Stein, P. C. (1943). Velocity Gradients and Internal Work in Fluid Motion. Journal of Boston Society of Civil Engineering, 30, 219-237.

Feder, J. (1988). Fractals. New York: Plenum. http://dx.doi.org/10.1007/978-1-4899-2124-6

Fei, X. (1994). Transport Hydraulics of Slurry and Granular Material. Beijing: Tsinghua University Press. (In Chinese)

Lu, S., Ding, Y., \& Guo, J. (1998). Kinetics of Fine Particle Aggregation in Turbulence. Advances in Colloid and Interface Science, 78, 197-235. http://dx.doi.org/10.1016/S0001-8686(98)00062-1

Wang, X., Shao, X., \& Li, D. (2002). Fundamentals of River Dynamics. Beijing: China Water and Power Press. (In Chinese)

Yang, T., Xiong X., Zhan X., \& Yang, M. (2003). On Flocculation of Cohesive Fine Sediment. Hydro-Science and Engineering, 2, 65-77. (In Chinese)

Zhu, Z., Yang, T., Zhao, M., \& Liang, C. (2009). Preliminary Study on the Critical Criterion for Distinguishing Floc Sedimentation and Gel-Like Network Sedimentation. Journal of Sediment Research, 1, 20-25. (In Chinese)

Zhu, Z. (2009). The Investigation on Phase Transition and the Influence of Shear on the Flocculation of Cohesive Sediment. M.S. Thesis, Beijing: Tsinghua University. (In Chinese) 